\title{
The Narrow-Line Regions of Seyfert and Radio Galaxies
}

\author{
Andrew S. Wilson \\ Space Telescope Science Institute, 3700 San Martin Drive, Baltimore, \\ $M D 21218, U S A$ \\ Astronomy Department, University of Maryland, College Park, MD \\ 20742, USA
}

\begin{abstract}
This paper reviews the morphology, excitation, and ionization of the gas in the narrow and extended narrow line regions (NLRs and ENLRs) in AGNs. Many high-resolution images of the NLRs of Seyfert galaxies are now available from $H S T$. In some galaxies, these images reveal straight, sharp-edged V-shaped structures (believed to be the projections of 3-dimensional cones), while in others there is a close association between the ionized gas and the radio components. The latter effect arises through radiative shocks driven into the surrounding interstellar gas by the outwardly moving radio components or jets. Photoionization models of ionization-bounded clouds are quite successful in reproducing the line intensity ratios, but a number of problems remain. These problems may be resolved if matter-bounded clouds are also present. Differences between the excitation levels of different NLRs and ENLRs may reflect variations in the fraction of matter-bounded clouds as well as variations in the ionization parameter. I discuss the issue of whether the radiation responsible for photoionizing the gas in Seyfert 2 galaxies originates from a hidden, compact type 1 Seyfert nucleus or from photoionizing shocks within the NLR. These two scenarios are hard to distinguish between. The photoionizing-shock model requires surprisingly high pre-shock densities, raising the question of whether the shocks are sufficiently energetic to power the observed line emission.
\end{abstract}

\section{Introduction}

Many active galaxies exhibit strong, highly excited emission lines with full widths at half maximum $\sim 200-1000 \mathrm{~km} \mathrm{~s}^{-1}$. The emitting gas has low density $\left(\sim 10^{2}\right.$ $10^{6} \mathrm{~cm}^{-3}$ ) and relatively large overall extent ( $10 \mathrm{pc}$ to $1 \mathrm{kpc}$ ). This spatial scale is loosely referred to as the narrow-line region (NLR). Extensive measurements of line intensities have been made (e.g., Koski 1978; Osterbrock 1993) and indicate that the gas is photoionized by radiation extending from the Lyman edge to at least soft X-rays with a hard spectrum (e.g., Ferland \& Osterbrock 1986). Unger et al. (1987) introduced the term 'extended narrow-line region' (ENLR) for high excitation gas which is farther from the nucleus than the NLR and emits narrow $\left(<50 \mathrm{~km} \mathrm{~s}^{-1}\right.$ ) lines. The ENLR is believed to be ionized by the same source of radiation as the NLR. 


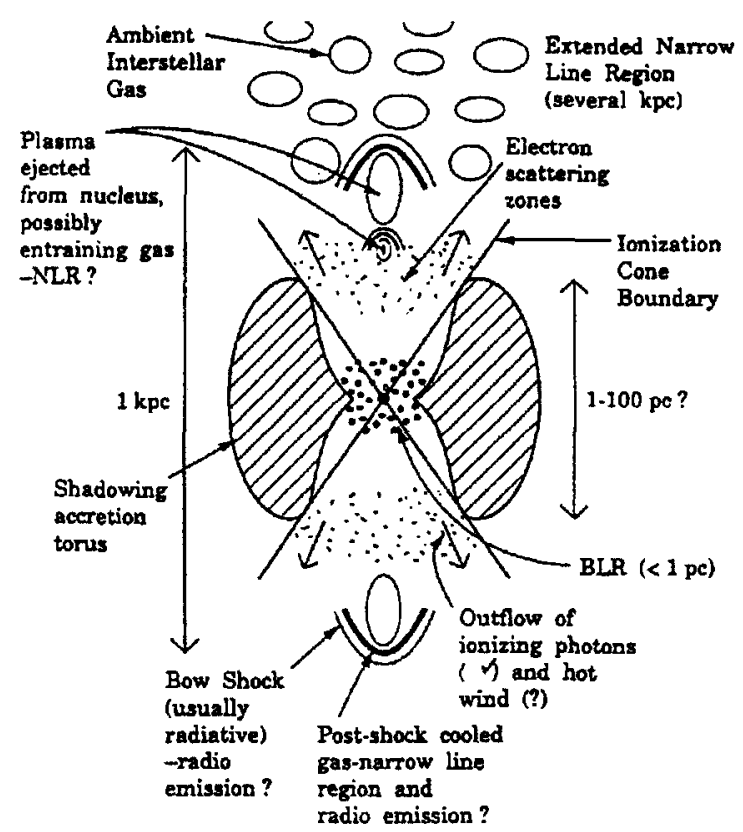

Figure 1. A schematic diagram of a Seyfert nucleus, showing currently recognized and probable components, and their typical size scales (from Wilson 1992).

Studies of the NLR and ENLR provide unique information about active nuclei for two main reasons. First, these regions may be spatially resolved, so key properties (density, velocity, ionization parameter, intensity of ionizing radiation, chemical abundance etc.) can be mapped directly. This contrasts with the situation for the broad-line region for which indirect means, such as reverberation mapping, must be used to measure spatial structure. Second, the NLR gas is of relatively low density so that many complexities inherent to photoionization modeling of the higher density $\left(10^{9}-10^{13} \mathrm{~cm}^{-3}\right)$ broad-line region (e.g., Rees, Netzer, \& Ferland 1989, and references therein) are absent.

In this article, I shall focus on three areas: the morphology of the NLR and its relation to the radio sources ( $(2)$, the excitation of the gas in the NLR and ENLR ( $\$ 3)$, and the nature of the source of ionizing photons ( $\$ 4)$. Previous recent reviews of extended, high-excitation gas in AGN include those of Whittle (1989), which focuses on the association between radio and optical line emission, and Wilson (1992), which emphasizes the evidence for anisotropic escape of ionizing radiation from the active nucleus.

\section{The Morphology of the Extended Gas: Results from HST}

Figure 1 is a cartoon of features recognized on the scales of the NLR and ENLR as of five years ago. It is believed that the nucleus is surrounded by a geometrically 
and optically thick (at least at optical and ultraviolet wavelengths) torus which obscures the broad-line region and other compact structures associated with the Seyfert 1 nucleus (Antonucci \& Miller 1985). The broad lines are rendered visible in polarized light by scattering off electrons or dust above and below the torus. The torus shadows the escaping ionizing radiation into two oppositely directed cones, which ionize gas in the NLR and ENLR, producing V-shaped emission-line projections (e.g., Pogge 1989; Tadhunter \& Tsvetanov 1989). Strong evidence that the collimation of the ionizing radiation is a result of geometric shadowing, rather than an intrinsically beamed process, is provided by Storchi-Bergmann, Mulchaey \& Wilson (1992), and by Kriss et al. (1997). Plasma, in the form of discrete 'plasmoids' or jets, is also ejected from the nucleus, generally along the rotation axis of the torus (Wilson \& Tsvetanov 1994). The radio emission may come from the plasmoid or jet itself (as in powerful radio sources), from the bow shock or from the post-bow shock cooling zone (Wilson \& Ulvestad 1987). Line emission may originate in ambient gas (Unger et al. 1987), gas entrained by the outflowing plasmoids or jets (e.g., De Young 1986; 1996), post-bow shock cooled gas (Pedlar, Dyson, \& Unger 1985; Taylor, Dyson, \& Axon 1992), or gas entrained in a hot, wide-angle outflowing wind (Krolik \& Begelman 1986).

The main development in this area over the last five years has been a rapidly growing number of high-resolution images in emission lines from the Hubble Space Telescope (HST). Most of these images have been obtained in three programs. The first is the Faint Object Spectrograph Investigation Definition Team observing program. About $20 \mathrm{AGN}$, including both Seyfert $1 \mathrm{~s}$ and $2 \mathrm{~s}$, have been observed (see Evans et al. 1994). Second, the Faint Object Camera Investigation Definition Team has observed mainly Seyfert 2 galaxies already known to have extended lines in ground-based observations (e.g., Capetti et al. 1996 and references therein). A similar criterion was used in the selection of objects for the third program, numbers GO-3724 and GO-5411/6332 (e.g., Bower et al. 1995). The main reason for selecting Seyfert $2 \mathrm{~s}$ for observation with the aberrated (preCOSTAR) telescope was to avoid the confusing effects of the PSF in Seyfert 1 galaxies, which are known to contain very bright, unresolved, nuclear continuum sources. There is thus a built-in bias in the HST imaging database of Seyfert galaxies towards Seyfert $2 \mathrm{~s}$ with extended emission lines. Further, the sensitivity to extended gas is worse in Seyfert 1s than Seyfert $2 \mathrm{~s}$ because of the confusing effects of the nuclear PSF (e.g., Bower et al. 1994). Because of these selection effects, great caution should be exercised in using the current $H S T$ archive for any statistical studies. In the following, I shall attempt to summaries the main results of these imaging studies.

\section{1. 'Ionization Cones'}

Images which show ionized gas with $\mathrm{V}$-shaped projections having sharp, straight edges are traditionally interpreted in terms of ionization of gas in the NLR or ENLR by a collimated, nuclear radiation field. The Seyfert 2 galaxy NGC 5643 exhibits a good example of such a structure. Figure 2 shows a map of the ratio [O III] $\lambda 5007 / \mathrm{H} \alpha+[\mathrm{N} \mathrm{II}] \lambda \lambda 6548,6583$ from $H S T$ images. In this case, the ionized gas is co-spatial with a jet-like radio source (Morris et al. 1985), and some of the structure seen within the $\mathrm{V}$-shaped envelope may be related to compression by the radio ejecta. The gas is likely being accelerated by the radio 


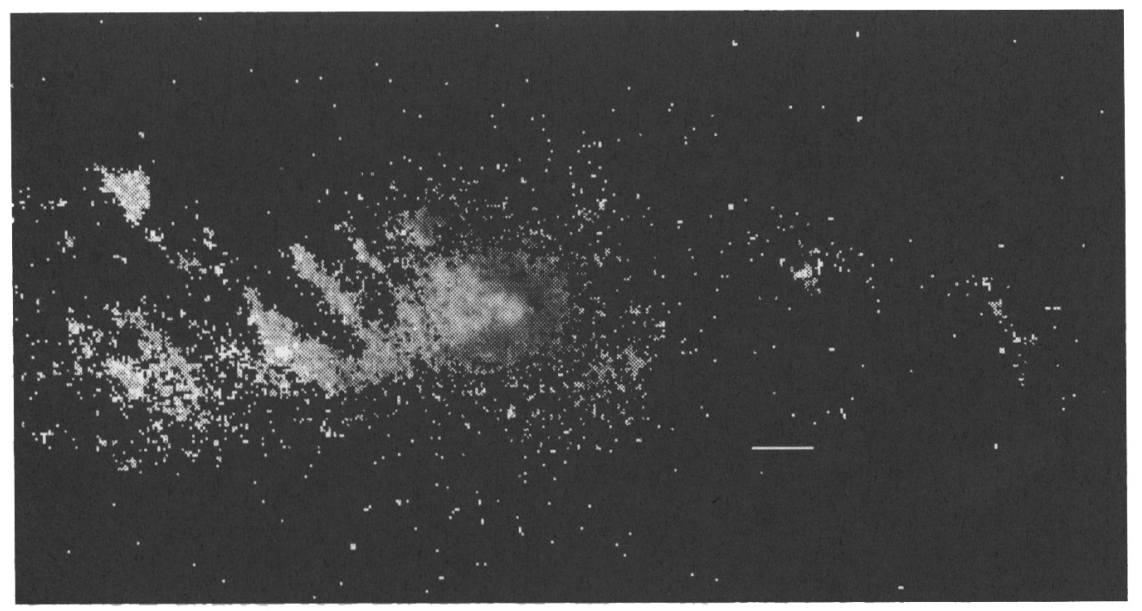

Figure 2. A map of the ratio [O $\mathrm{HI}] \lambda 5007 / \mathrm{H} \alpha+[\mathrm{N} \mathrm{II}] \lambda \lambda 6548,6583$ in the type 2 Seyfert galaxy NGC 5643 (from Simpson et al. 1996). This ratio is sensitive to both gaseous excitation and reddening. Highexcitation gas is light, low-excitation dark; the lowest-excitation gas is not shown. The white bar indicates $1^{\prime \prime}(\approx 78 \mathrm{pc})$. Note the 'pacmanshaped' region of low-excitation or significantly reddened gas immediately to the north, west and south of the apex of the V-shaped projection.

ejecta and it is possible that ionizing radiation from photoionizing shocks (cf. Dopita \& Sutherland 1995), as well as from the putative compact nuclear source, contributes to the ionization of the gas. However, the sharp, straight edges are hard to understand in a model of a turbulent, outflowing and entraining jet or wind ionized by an isotropic nuclear source or 'in situ' by shocks. Other examples of ionization cones imaged by HST include NGC 1068 (Evans et al. 1991; Macchetto et al. 1994), NGC 4151 (Evans et al. 1993), and NGC 5728 (Wilson et al. 1993).

The properties of ionization cones have recently been discussed elsewhere (Wilson 1996), so I will confine myself to a brief summary of the main points.

- Both bi-cones and single (one-sided, like Fig. 2) cones are found. In all known one-sided cases, the observed cone projects against the far side of the galaxy disk, suggesting that a counter-cone may be present but hidden by obscuration in the disk.

- NGC 5252 is the best example of a collimated radiation field illuminating gas which is apparently undisturbed by the nuclear activity (Tadhunter \& Tsvetanov 1989). Prieto \& Freudling (1993) have mapped the distribution of $\mathrm{HI}$ in the $21 \mathrm{~cm}$ line and find that the neutral hydrogen tends to avoid the ionization cones and that there is velocity continuity from neutral to ionized gas. These results confirm the interpretation that the ionized arcs within the $\mathrm{V}$-shaped envelopes represent pre-existing ring structures that have been ionized by a collimated, nuclear radiation field (i.e. the optical 
nebulosity is ionization-bounded). Unfortunately, most other 'cones' are too compact for the distribution of neutral hydrogen to be mapped in this way.

- Projection effects are expected to modify both the apparent opening angle and incidence of ionization cones. If the illuminated gas is distributed in a sphere or spheroid, V-shaped projections are expected in only Seyfert $2 \mathrm{~s}$ (for the simplest picture in which the torus blocks all radiation from the inner nucleus). The observed opening angle of the V-shaped emission-line structure is equal to the opening angle of the radiation cone only when the cone's axis is in the plane of the sky. For other inclinations, the observed opening angle is larger than the true opening angle of the radiation cone. These projection effects are different if the radiation cone illuminates a thin disk with center at the cone's apex. In this case, V-shaped emission line projections are expected in both Seyfert $1 \mathrm{~s}$ and $2 \mathrm{~s}$ and the observed opening angle can be larger or smaller than the true opening angle. For a detailed discussion and simulations of these effects, see Mulchaey (1994) and Mulchaey, Wilson, \& Tsvetanov (1996).

- There is no clear relationship between the axes of the cones and those of the host-galaxy disks.

- The radio axis is, in general, tightly aligned with the axis of the cone. This can occur even when the radio source is much smaller than the V-shaped emission-line projections (e.g., Wilson \& Tsvetanov 1994), indicating that, in at least these cases, the alignment results from collimation by co-planar gas disks, rather than direct compression or excitation by the radio ejecta. In a few cases, such as NGC 4151, there is a misalignment between the hundred-parsec scale radio jet and the cone axis. Such misalignments may result from projection effects, in which the V-shaped nebulosity arises through an ionizing photon cone grazing a gas disk, with the radio jet coincident with the cone axis (Pedlar et al. 1993; Robinson et al. 1994).

- A number of V-shaped structures contain high-velocity, outflowing gas, as indicated by line splitting in spectroscopy from the ground. Examples include NGC 3227 (Mundell et al. 1995), NGC 3281 (Storchi-Bergmann, Wilson, \& Baldwin 1992), and NGC 4051 (Christopoulou et al. 1996). The resolution of the emission-line images from the ground is not good enough to see whether the edges are sharp and straight, as expected in the geometrical shadowing model. In such cases, it is not clear that the ionized nebulosity is defined by a collimated nuclear radiation field. It is possible that edge-brightened, V-shaped nebulosities could result from entrainment and compression of ambient gas by an expanding wind, with the nebulosity being matter-bounded, rather than ionization-bounded. There may be a whole continuum of situations giving V-shaped projections, ranging from those in which ambient, kinematically undisturbed gas is illuminated by a collimated radiation field, to matter-bounded cases in which compression of ambient gas by an outflowing wind is the dominant effect shaping the nebulosity. 

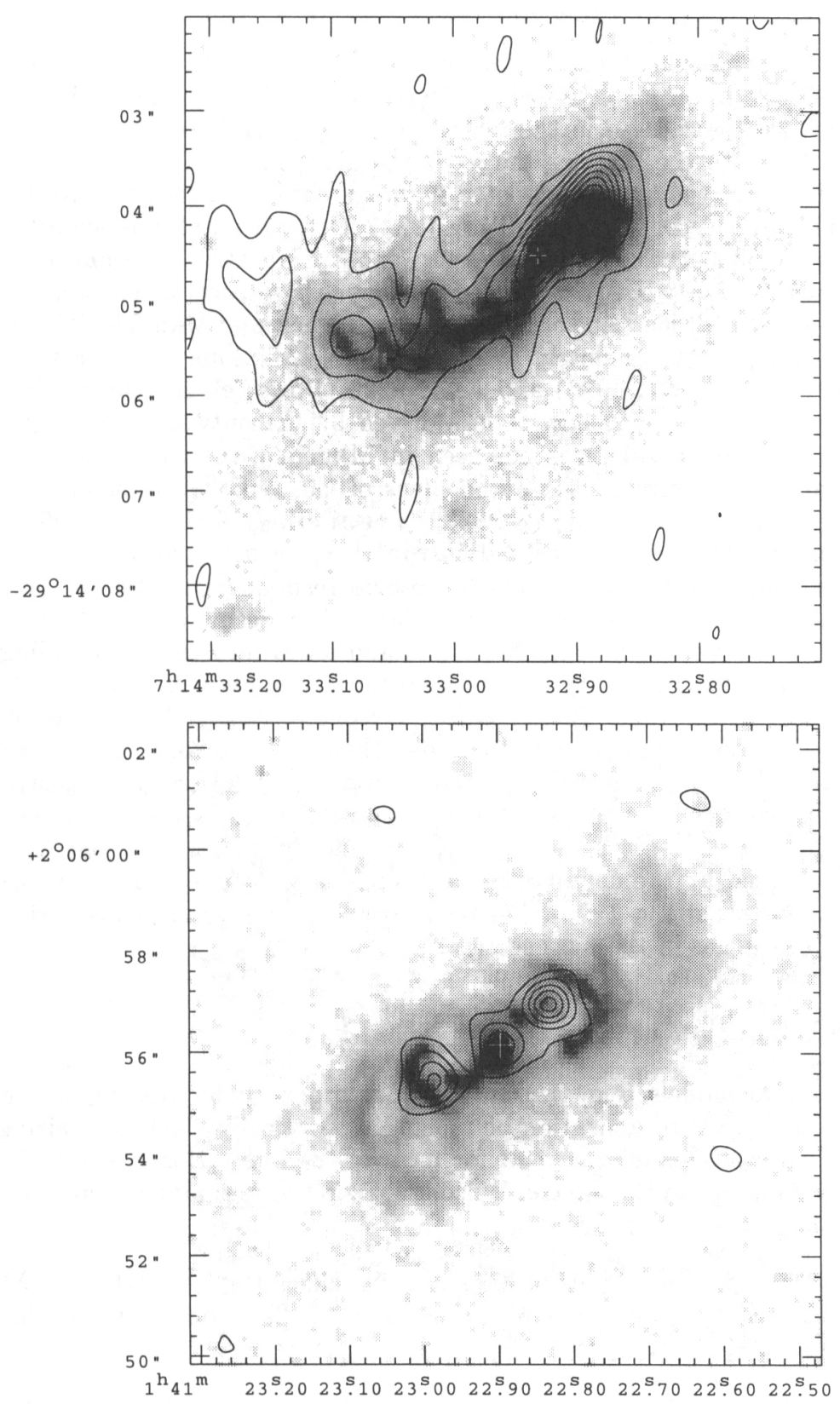

Figure 3. Top: The $\mathrm{H} \alpha+\left[\mathrm{N}_{\mathrm{II}}\right] \lambda \lambda 6548,6583$ emission of the Seyfert 2 galaxy ESO $428-\mathrm{G} 14$, based on an HST, image is shown in grey scale. Contours of the $6 \mathrm{~cm}$ radio emission are overlaid (from Falcke et al. 1996). Bottom: $H S T \mathrm{H} \alpha+\left[\mathrm{N} \mathrm{II}_{\mathrm{H}}\right] \lambda{ }^{2} 6548,6583$ and VLA radio images of Mkn 573 in the same format as above (from Wilson, Falcke, \& Simpson, in preparation). 


\subsection{Jets, Lobes, and Bow Shocks}

In many Seyfert galaxies, the ionized gas is closely associated with the radio jets and lobes. A recent example is shown in the upper panel of Fig. 3. In this galaxy, the ionized gas in the NW part of the jet follows a 'figure of eight' structure, while to the SE long curved strands are seen. These morphologies are reminiscent of the helically twisted filaments found in the jet of NGC 4258 (Ford et al. 1986; Cecil, Wilson, \& Tully 1992). Other examples of Seyfert NLRs which closely follow the radio jets include Mkn 3 (Capetti et al. 1995, 1996; see also Pogge 1997), in which the ionized gas appears to form a 'cocoon' around the radio jet, Mkn 1066 (Bower et al. 1995) and perhaps Mkn 348 (Capetti et al. 1996). The NLRs are closely associated with radio-lobe structures in Mkn 78 (Capetti et al. 1994, 1996) and NGC 5929 (Bower et al. 1994). Features apparently associated with bow shocks driven by the outwardly moving radio lobes into the surrounding gas are seen in Mkn 573, in which shells of line emission appear to envelop the outer edges of the radio components (Fig. 3, lower panel; Pogge \& De Robertis 1995; Capetti et al. 1996; Pogge 1997), and possibly in NGC 3393 (Pogge 1997; Baldwin et al., in preparation).

It has long been believed that the close correspondence between the radio and emission-line structures in Seyfert galaxies is a result of radiative shocks driven by the outwardly moving radio jets and lobes into the surrounding gas (e.g., Heckman et al. 1982; Wilson \& Ulvestad 1983, Pedlar, Dyson, \& Unger 1985; Whittle et al. 1986, 1988; Taylor, Dyson, \& Axon 1992). The gas is initially heated to a high temperature, but then cools to form a thin shell or sheath around, or mixed in with, the relativistic gas. The HST images strongly support this scenario. A currently more controversial issue is whether the cooled gas is maintained in an ionized state at $\sim 10^{4} \mathrm{~K}$ through photoionization by radiation emitted by the compact Seyfert nucleus or by the shocks themselves (cf. Dopita \& Sutherland 1995). I shall return to this issue after discussing the excitation of the gas in the next section.

\section{The Excitation of the NLR and ENLR}

Photoionization models (e.g., Ferland \& Osterbrock 1986; Binette, Robinson, \& Courvoisier 1988) of ionization-bounded (i.e. optically thick to ionizing radiation) clouds successfully account for most of the line ratios in the NLR and ENLR. As summarized by Binette, Wilson, \& Storchi-Bergmann (1996, hereafter BWSB), three problems remain:

1. The strengths of high-excitation lines, such as [Nev] $\lambda 3426, \mathrm{C}$ IV $\lambda 1549$, $\mathrm{CIII}] \lambda 1909$, and $[\mathrm{FeVII}] \lambda 6087$, predicted by the models are too low by factors of 5-15.

2. The temperature-sensitive flux ratio $R([\mathrm{O}$ III $])=I(\lambda 4363) / I(\lambda 5007)$ yields higher electron temperatures than prediced by the models. Photoionization models of ionization-bounded clouds with solar abundances pre$\operatorname{dict} R([\mathrm{O} \mathrm{III}]) \leq 0.01$ (i.e., $\left.T_{e} \leq 11,000 \mathrm{~K}\right)$, while observations indicate $0.01 \leq R([\mathrm{O} I 1 \mathrm{II}]) \leq 0.03$ (i.e., $11,000 \leq T_{e} \leq 20,000 \mathrm{~K}$; Tadhunter, Robinson, \& Morganti 1989). 


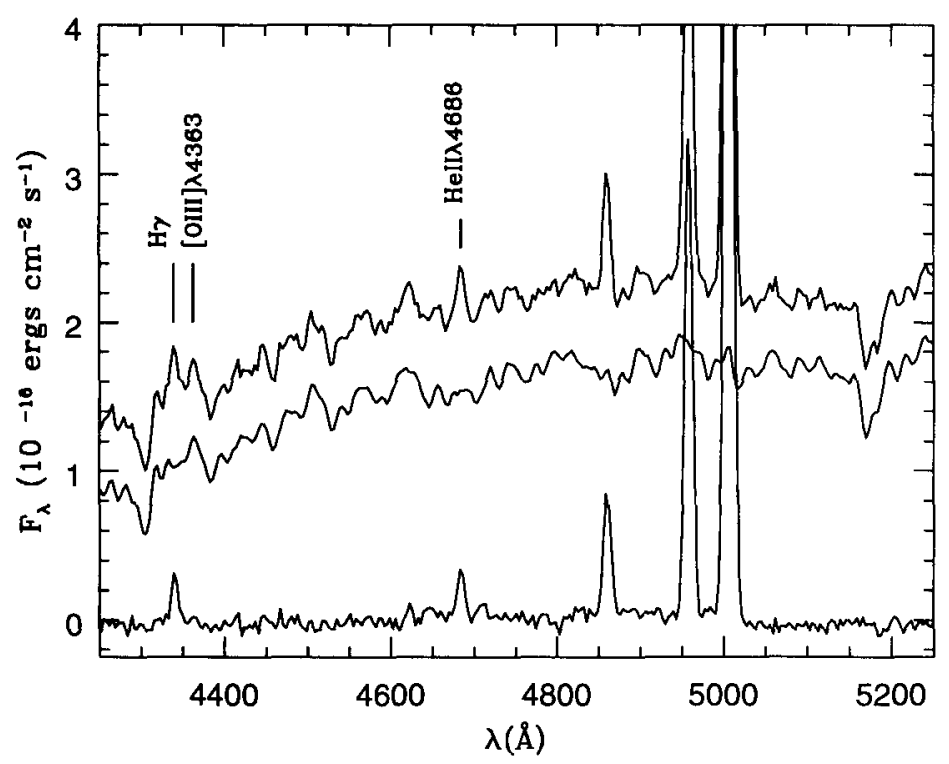

Figure 4. Illustration of the effects of an underlying old stellar population on the detectability of the emission lines [O $\left.{ }_{11}\right] \lambda_{4} 4363$ and He II $\lambda 4686$ (from Storchi-Bergmann et al. 1996). Top: Nuclear spectrum of the radio galaxy PKS 0634-206. Middle: Stellar population template from the bulge of NGC 1097 with an arbitrary vertical shift for clarity. Bottom: Difference between nuclear and stellar population spectra. [O III] $\lambda 4363$ is not detected in the nucleus of PKS 0634-206.

3. In models with ionization-bounded clouds, the $I(\mathrm{He} I \mathrm{I} \lambda 4686) / I(\mathrm{H} \beta)$ ratio is determined by the slope of the ionizing continuum between 13.6 and $54.4 \mathrm{eV}$, and is sensibly independent of the ionization parameter $U$, defined as the ratio of the density of impinging ionizing photons to the density of the gas cloud. The ratio predicted by these models for canonical continuum shapes is often much less than that observed. of:

Solutions which have been proposed for these problems include the presence

1. Shock waves (e.g., Contini \& Viegas-Aldrovandi 1989; Sutherland, Bicknell, \& Dopita 1993; Dopita \& Sutherland 1995) or cosmic rays (e.g., Ferland \& Mushotzky 1984; Viegas-Aldrovandi \& Gruenwald 1988), both of which may provide additional heating and increase $R\left(\left[\begin{array}{ll}\mathrm{III}\end{array}\right]\right.$ ).

2. An ionizing continuum which is photoelectrically cut-off at soft X-ray wavelengths. The effect of such a hard ionizing spectrum is to increase the relative strengths of high-ionization lines (e.g., He II $\lambda 4686$, [Ne v] $\lambda 3426$, and $[\mathrm{FeVII}] \lambda 6087)$ through the increased ionizing flux at and above their ionization energies (e.g., Ferland \& Mushotzky 1982; Evans \& Dopita 1986; Bergeron et al. 1989). 


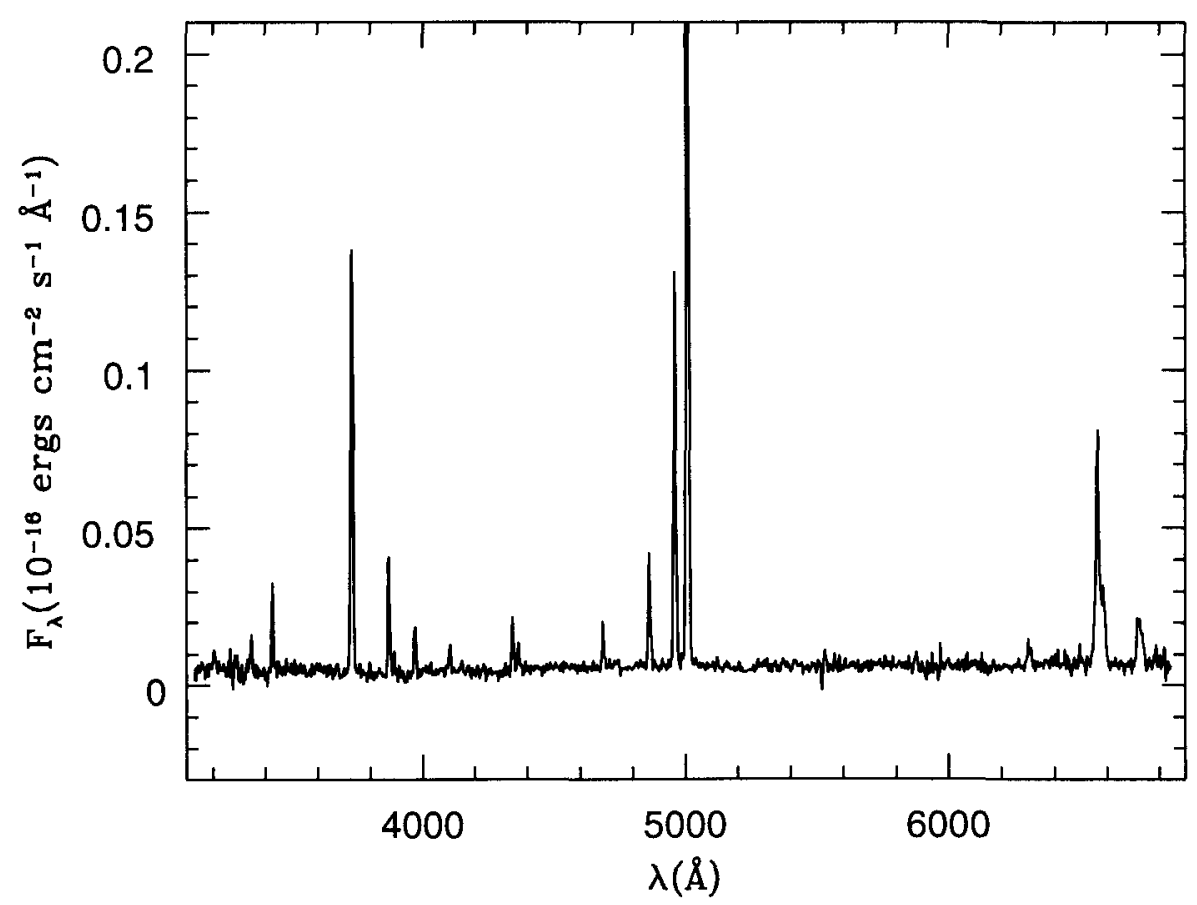

Figure 5. The spectrum of gas $11^{\prime \prime} \mathrm{SW}$ of the nucleus of the radio galaxy PKS 0349-278 (from Storchi-Bergmann et al. 1996). The contribution of the stellar population is negligible.

3. High-density gas, which can increase $R([\mathrm{O} \mathrm{III}])$. This may be a plausible explanation for the compact NLR, but the required densities $\left(n_{e} \geq 10^{5} \mathrm{~cm}^{-3}\right)$ are much too high for spatially extended gas ( $\geq 100 \mathrm{pc}$ scale).

4. Low-metallicity gas, which tends to increase $R([\mathrm{O}$ III $])$. However, sub-solar metallicity is implausible in the inner regions of Seyfert galaxies, which are mostly early-type spirals.

5. Matter-bounded clouds, which increases the strengths of high-ionization lines and the He II $\lambda 4686 / \mathrm{H} \beta$ ratio (Viegas-Aldrovandi 1988; Viegas \& Prieto 1992).

In order to measure the strengths of weak diagnostic lines in low density gas, we have recently obtained high $S / N$ ratio optical spectra of spatially extended, high-excitation nebulosities in Seyfert and radio galaxies (Storchi-Bergmann et al. 1996). We have tried to obtain accurate flux measurements over a wide wavelength range (through alignment of the slit with the parallactic angle), reliable deblending of [ $\mathrm{O}$ III $] \lambda 4363$ and $\mathrm{H} \gamma$ (through adequate spectral resolution, i.e., $8 \AA$ ) and measurements of weak emission lines (through subtraction of the spectrum of the stellar population, when present). The effects of contamination by starlight on the strength of [O III] $\lambda 4363$ is illustrated in Fig. 4. The spectra of 


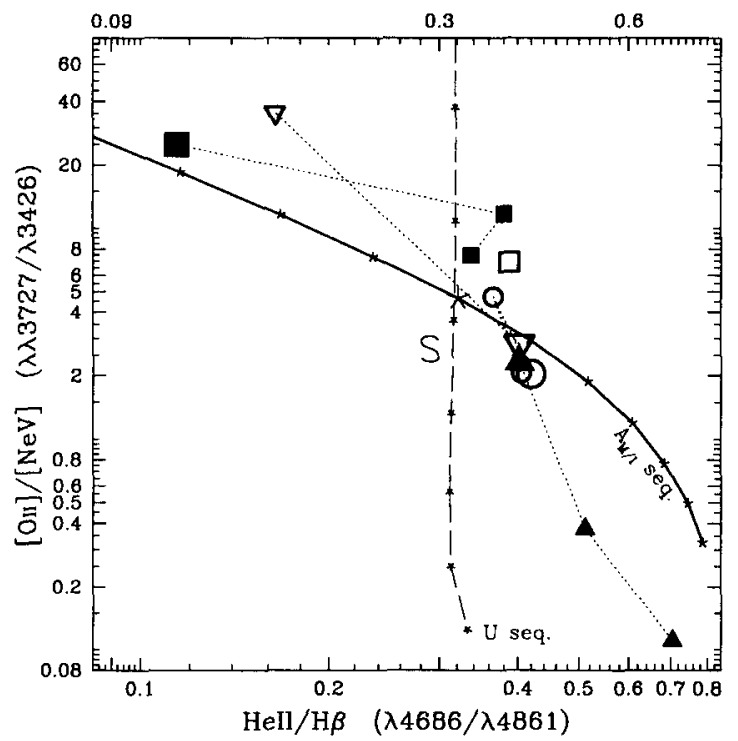

Figure 6. Diagram of the line ratio $\left[\mathrm{O}_{\mathrm{II}}\right] \lambda 3727 /[\mathrm{Ne}$ V $] \lambda 3426$ against He II $\lambda 4686 / H \beta$ (from Binette, Wilson, \& Storchi-Bergmann 1996). The symbols represent the measurements of five Seyfert and radio galaxies by Storchi-Bergmann et al. (1996). Larger symbols denote the nuclear values and dotted lines join measurements at different locations in the same galaxy. Filled symbols represent radio galaxies. The letter ' $S$ ' indicates the position of a 'mean' Seyfert nucleus. The solid line represents the $A_{M / I}$ sequence $\left(0.04 \leq A_{M / I} \leq 16\right)$, with $A_{M / I}$ increasing from left to right. The asterisks are separated by $0.2 \mathrm{dex}$ in $A_{M / I}$, with the larger asterisk denoting the model with $A_{M / I}=1$. The long dashed line represents the traditional $U$ sequence, with a separation in $U$ of 0.2 dex between asterisks. The range covers $4 \times 10^{-4} \leq U \leq 10^{-1}$ and extends beyond the figures limits.

late-type stellar populations include an apparent peak near $4363 \AA$. If the stellar spectrum is not subtracted, this feature may be mistaken for [O $\mathrm{III}] \lambda 4363$ and the temperature grossly overestimated.

An example of the results is shown in Fig. 5. This off-nuclear spectrum is of very high excitation, with neutral and lowly ionized species weak or absent, and is best interpreted in terms of matter-bounded clouds photoionized by a hard continuum. In general, the results of Storchi-Bergmann et al. (1996) reaffirm the three problems listed above for photoionization models with ionization-bounded clouds. In particular, we confirm the range of electron temperatures found by Tadhunter, Robinson, \& Morganti (1989). We suspect that some reported cases of $R([\mathrm{OHI}])>0.03\left(T_{e}>19,000 \mathrm{~K}\right)$ result from inadequate deblending of $[\mathrm{O} \mathrm{III}] \lambda 4363$ from $\mathrm{H} \gamma$ and/or failure to subtract properly the spectrum of the stellar population. 

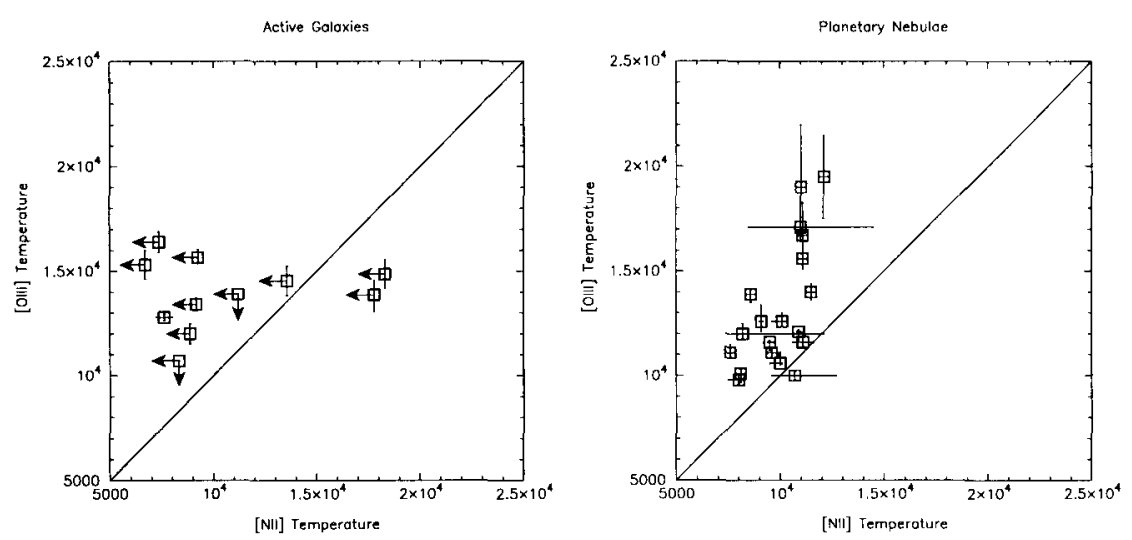

Figure 7. Left: A plot of [O III] temperature versus [N II] temperature (in $\mathrm{K}$ ) for nuclear and extended gas in active galaxies using the data of Storchi-Bergmann et al. (1996). The temperature sensitive [N II] $\lambda 5755$ line is detected in only the nucleus of $\mathrm{Mkn} 573$, so the [N $\mathrm{NI}]$ temperatures are upper limits at all other positions. Right: A similar plot for planetary nebulae, using the temperatures listed by Kingsburgh \& Barlow (1994). It is notable that the range of $T_{e}\left(\left[\mathrm{O}_{\mathrm{III}}\right]\right)$ is similar in the two types of object and that there is a trend for $T_{e}([\mathrm{O} \mathrm{III}])>T_{e}([\mathrm{~N} \mathrm{II}])$ in both types.

Spectra like Fig. 5 indicate that matter-bounded clouds are present in the NLR and ENLR. Our results favor a resolution of the 'three problems' along the lines suggested by Viegas \& Prieto (1992). BWSB argue in favor of two populations of ionized clouds in extended emission-line regions - a matter-bounded component responsible for most of the He II and high-excitation forbidden-line emission, and an ionization-bounded component responsible for low- to intermediate-ionization forbidden lines. BWSB propose that variations in apparent excitation in active galaxies represent variations in the relative contributions of matter- and ionization-bounded clouds. A new series of photoionization calculations can then be made by varying the parameter $A_{M / I}$, which is defined as the ratio of the solid angles subtended at the ionizing source by the matter- and ionization-bounded clouds. This $A_{M / I}$ sequence is found to provide a much better representation of various line ratios than the traditional $U$ sequence with ionization-bounded clouds. For example, the $A_{M / I}$ sequence provides a natural explanation for newly found correlations (BWSB) between various measures of excitation from forbidden-line ratios (e.g., $[\mathrm{O} \mathrm{III}] \lambda 5007 /[\mathrm{O} \mathrm{II}] \lambda 3727,[\mathrm{Ne} \mathrm{V}] \lambda 3426 /[\mathrm{O} \mathrm{II}] \lambda 3727$, and $[\mathrm{Ne} \mathrm{V}] \lambda 3426 / \mathrm{H} \beta)$ and the $\mathrm{He}$ II $\lambda 4686 / \mathrm{H} \beta$ ratio (e.g., Fig. 6). These correlations cannot be explained within the framework of the widely accepted $U$ sequence among ionizationbounded clouds, since the $\mathrm{He}$ II $\lambda 4686 / \mathrm{H} \beta$ ratio is almost independent of $U$ (Fig. $6)$.

Furthermore, the temperature problem can be solved in the $A_{M / I}$ sequence provided the thickness and ionization parameter $\left(U_{M B} \geq 0.04\right)$ of the matter- 
bounded clouds are appropriately selected. The key to obtaining sufficiently high temperatures in pure photoionization models is that the matter-bounded clouds must be sufficiently thin that their temperature is governed by photoionization of $\mathrm{He}^{+}$, but sufficiently thick that they contribute to the [O III] lines (BWSB). Thinner clouds would have high temperatures but little [O III] emission, while thicker clouds would emit more strongly in [O III], but the temperature would be too low. The required thickness of the matter-bounded clonds is such that $\sim 35-40 \%$ of the ionizing photons are absorbed. This thickness coincides with a peak in the effective heating rate (see Fig. 2 of BWSB). BWSB speculate that such clouds form naturally if the matter-bounded clouds represent a low-density wind driven off ambient interstellar clouds by the intense radiation ( $\mathrm{cf}$. Bertoldi \& McKee 1990). In this picture, the ionization-bounded component corresponds to the dense core of the cloud.

The $A_{M / I}$ sequence also provides much stronger high-excitation lines (e.g., [Nev] $\lambda 3426, \mathrm{C}$ IV $\lambda 1549$ ) than the $U$ sequence, in accordance with observations. Lastly, the $A_{M / I}$ sequence predicts a difference of $\sim 5000 \mathrm{~K}$ between the values of $T_{e}$ derived from $R([\mathrm{O} \mathrm{III}])$ and $R([\mathrm{NII}])(R([\mathrm{NII}])=I(\lambda 5755) / I(\lambda 6583))$, because $[\mathrm{N} \mathrm{II}]$ originates in the cooler, ionization-bounded clouds. Figure 7 shows that, indeed, $T_{e}([\mathrm{O} \mathrm{III}])>T_{e}([\mathrm{~N} \mathrm{II}])$ and that a similar trend is seen in planetary nebulae. More generally, the ranges of $T_{e}([\mathrm{O}$ III $]$ ) seen in AGN ENLRs and planetary nebulae are similar. This result argues against the notion that heating by shocks or relativistic particles is important in AGN ENLRs.

\section{The Source of Ionizing Photons: Compact Nucleus or Photoion- izing Shocks?}

The NLRs and ENLRs of Seyfert galaxies and FR II radio galaxies are traditionally interpreted in terms of photoionization of gas by a compact nuclear source. Dopita \& Sutherland (1995) have argued instead that the ionizing photons originate in 'photoionizing shocks' of velocity $300-500 \mathrm{~km} \mathrm{~s}^{-1}$ within the NLR itself. In this latter model, Seyfert galaxies are cases in which emission from the photoionized precursor or photoionized cooling region dominates that from collisionally ionized gas behind the shock.

The results described in $\S 2$ show clearly that radiative shocks are present in the NLRs of Seyfert galaxies and must emit ionizing radiation at some level. It is also clear that many Seyfert $2 \mathrm{~s}$ contain 'hidden' Seyfert 1 nuclei, which are known to harbor a compact (sub-parsec), luminous source of ionizing photons. Thus the question is not whether fast radiative shocks exist in the NLR, but rather whether their ionizing radiation dominates that from the compact nucleus. How can we resolve this issue?

Here are some parameters which do not, in my view, distinguish between photoionization by a central source and photoionization 'in situ' by shocks:

1. The electron temperature. Models of Seyfert-type emission spectra with ionization-bounded clouds predict temperatures $T_{e}<11,000 \mathrm{~K}$ for solar abundances whether the ionizing photons originate in a shock (see Fig. 7 of Dopita \& Sutherland 1995) or the central source (Tadhunter, Robinson, \& Morganti 1989; BWSB). In both cases, matter-bounded clouds are 
needed to give [O $\mathrm{III}]$ temperatures up to $\sim 18,000 \mathrm{~K}$. Ultraviolet temperature diagnostics, such as $\mathrm{C}_{111} \lambda 977 / \lambda 1909$ and $\mathrm{N}$ III $\lambda 990 / \lambda 1750$ (Kriss et al. 1992) are ambiguous because dielectronic recombination and continuum fluorescence may be strong contributors to C III $\lambda 977$ and $\mathrm{N}$ III $\lambda 990$ (Ferguson, Ferland, \& Pradhan 1995).

2. The spatial variation of excitation within the NLR. In both models, such variations depend on the run of electron density and the fraction of matterbounded clouds with position, neither of which can be predicted a priori.

3. The strengths of high-excitation UV lines, such as CIV $\lambda 1549$. Strong emission from these lines can be interpreted in terms of either photoionized matter-bounded clouds (BWSB) or emission from the cooling zone of a shock (Dopita \& Sutherland 1995).

4. A correlation between excitation level and velocity spread, either spatially within one galaxy or between different galaxies. If the gas is accelerated by shocks, regions of low density will tend to have high velocities and those of high density low velocities, thanks to the expected similar pressures $\sim \rho V^{2}$. Thus gas with high velocity should have a higher ionization parameter than gas with low velocity, whatever the source of ionizing photons.

5. The existence of 'energy deficits' in Seyfert 2 galaxies. In these galaxies, there appear to be too few ionizing photons to account for the observed recombination-line emission. What is usually done is to extrapolate the observed nuclear optical or UV continuum spectrum (e.g., Kinney et al. 1991) to estimate the number of ionizing photons available, and then compare with the observed number of hydrogen recombination photons. The deficit so found is generally taken to imply that our view of the source of ionizing photons is blocked, but that the gas sees this source relatively unattenuated. If the gas is ionized by shocks on the $100 \mathrm{pc}$ scale, which is larger than the 'vertical' thickness of the obscuring torus, then the UV emission from the shocks should be little attenuated by the torus and an 'energy deficit' should not exist. However, a large part of the ionizing radiation from a $300-500 \mathrm{~km} \mathrm{~s}^{-1}$ shock is emitted as line emission in the EUV. In fact, the UV continuum flux near $1300 \AA$ from a $400 \mathrm{~km} \mathrm{~s}^{-1}$ shock is $\sim 100$ times lower than that from a power-law spectrum containing the same number of ionizing photons (Morse, Raymond, \& Wilson 1996). Thus photoionizing shocks can 'hide' large fluxes beyond the Lyman limit, even when they are 'out in the open', beyond the obscuring torus (see Morse, Raymond, \& Wilson 1996 for further discussion of this issue).

A more modest goal is to check whether photoionizing shocks are energetically capable of powering the NLR, given the known luminosities in emission lines from Seyfert galaxies. Previous discussions of such 'kinetic energy' models for the NLR (Wilson, Ward \& Haniff 1988; Whittle 1989) have been hampered by poor knowledge of the true scale of the NLR, and hence the area of the shocks. Mkn 573 is one of the clearest examples among Seyfert galaxies of radio lobes driving radiative shocks into the surrounding gas (Fig. 3, lower panel). In this object, we may derive the shock areas and emission-line luminosities directly 
from the $[\mathrm{O} \mathrm{III}] \lambda 5007$ image. For $H_{0}=75 \mathrm{~km} \mathrm{~s}^{-1} \mathrm{Mpc}^{-1}$, the $[\mathrm{O}$ III $] \lambda 5007$ luminosities of the two 'arcs' wrapped around the outer sides of the radio lobes are $L([\mathrm{O} \mathrm{III}])=9.2 \times 10^{40}$ and $8.0 \times 10^{40} \mathrm{ergs} \mathrm{s}^{-1}$. The total emission-line luminosity $L_{E}$ for a Seyfert-type spectrum is $\sim 14 L([\mathrm{O} \mathrm{III}])$, corresponding to $1.3 \times 10^{42}$ and $1.1 \times 10^{42} \mathrm{ergs} \mathrm{s}^{-1}$, respectively. I assume the emission lines outline the shocks (i.e., small cooling lengths) and that the shocks have the same extent along our lines of sight as in the plane of the sky, and hence derive shock areas of $A \approx 4.0 \times 10^{42}$ and $1.0 \times 10^{42} \mathrm{~cm}^{2}$, respectively. Assuming a shock velocity of $V=300 \mathrm{~km} \mathrm{~s}^{-1}$ (comparable to the emission line widths), we may calculate the required pre-shock particle number densities from $n=2 L_{E} / \epsilon A m V^{3}$, where $\epsilon$ is the efficiency with which the shock power is converted into emissionline luminosity. The results are $n=12 / \epsilon$ and $41 / \epsilon \mathrm{cm}^{-3}$. Given that $\epsilon$ is expected to be a few tenths assuming a $100 \%$ covering factor (e.g., Dopita \& Sutherland 1995), these densities are high, but perhaps not outrageous, for a few hundred parsecs from the nucleus of an early-type spiral; they would be lower for a higher shock velocity. Taking the average of these two densities, the ram pressure is $\rho V^{2}=4.8 \times 10^{-8} / \epsilon \mathrm{erg} \mathrm{cm}^{-3}$. If the shock is driven by the pressure of relativistic particles and magnetic fields in the radio lobes, we require $B=8 \times 10^{-4} / \epsilon^{1 / 2}$ Gauss (assuming equipartition between cosmic rays and magnetic fields), which is fully an order of magnitude larger than the lower limit (the radio lobes are only marginally resolved) to the equipartition magnetic field calculated from the radio emission. This suggests that the radio lobes may not have enough pressure to drive shocks of the power required to account for the line emission. If the emission of the entire NLR is supposed to be powered by these two shocks, the above pre-shock densities must be multiplied by a factor of $\sim 4$. Thus, to be energetically viable, photoionizing shock models require uncomfortably high pre-shock densities in Mkn 573.

Acknowledgments. I thank my collaborators, especially L. Binette, G. A. Bower, H. Falcke, J.A. Morse, J.S. Mulchaey, C. Simpson, and T. StorchiBergmann.

\section{References}

Antonucci, R. R. J., \& Miller, J.S. 1985, ApJ, 297, 621.

Bergeron, J., Petitjean, P., \& Durret, F. 1989, A\&A, 213, 61.

Bertoldi, F., \& McKee, C. F. 1990, ApJ, 354, 529.

Binette, L., Robinson, A., \& Courvoisier, T.J.-L. 1988, A\&A, 194, 65.

Binette, L., Wilson, A.S. \& Storchi-Bergmann, T. 1996, A\&A, 312, 365.

Bower, G.A., Wilson, A.S., Mulchaey, J.S., Miley, G.K., Heckman, T.M., \& Krolik, J. H. 1994, AJ, 107, 1686.

Bower, G.A., Wilson, A.S., Morse, J.A., Gelderman, R., Whittle, M., \& Mulchaey, J. S. 1995, ApJ, 454, 106.

Capetti, A., Macchetto, F.D., Sparks, W. B., \& Boksenberg, A. 1994, ApJ, 421, 87.

Capetti, A., Macchetto, F.D., Axon, D.J., Sparks, W. B., \& Boksenberg, A. 1995, ApJ, 448, 600 . 
Capetti, A., Axon, D.J., Macchetto, F.D., Sparks, W.B., \& Boksenberg, A. 1996, ApJ, in press.

Cecil, G., Wilson, A.S., \& Tully, R. B. 1992, ApJ, 390, 365.

Christopoulou, P.E., et al. 1996, MNRAS, in press.

Contini, M., \& Viegas-Aldrovandi, S. M. 1989, ApJ, 343, 78.

De Young, D. S. 1986, ApJ, 307, 62.

De Young, D.S. 1996, in Energy Transport in Radio Galaxies and Quasars, eds. P. E. Hardee, A. H. Bridle, \& J.A. Zensus, ASP Conference Series Vol. 100, (San Francisco: Astronomical Society of the Pacific) p. 261.

Dopita, M.A., \& Sutherland, R. S. 1995, ApJ, 455, 468.

Evans, I. N., \& Dopita, M. A. 1986, ApJ, 310, L15.

Evans, I. N., Ford, H. C., Kinney, A. L., Antonucci, R. R. J., Armus, L.,\& Caganoff, S. 1991, ApJ, 369, L27.

Evans, I. N., Tsvetanov, Z., Kriss, G. A., Ford, H.C., Caganoff, S., \& Koratkar, A. P. 1993, ApJ, 417, 82.

Evans, I. N., Ford, H.C., Kriss, G.A., \& Tsvetanov, Z. 1994, in The First Stromlo Symposium: The Physics of Active Galaxies, eds. G. V. Bicknell, M.A. Dopita, \& P. J. Quinn, ASP Conference Series Vol. 54, (San Francisco: Astronomical Society of the Pacific) p. 3.

Falcke, H., Wilson, A.S., Simpson, C., \& Bower, G. A. 1996, ApJ, in press.

Ferguson, J.W., Ferland, G. J., \& Pradhan, A. K. 1995, ApJ, 438, L55.

Ferland, G.J., \& Mushotzky, R. F. 1982, ApJ, 262, 564.

Ferland, G.J., \& Mushotzky, R. F. 1984, ApJ, 286, 42.

Ferland, G. J., \& Osterbrock, D. E. 1986, ApJ, 300, 658.

Ford, H. C., Dahari, O., Jacoby, G. H., Crane, P.C., \& Ciardullo, R. 1986, ApJ, 311, L7.

Heckman, T.M., Miley, G.K., Balick, B., van Breugel, W.J.M., \& Butcher, H. R. 1982, ApJ, 262, 529.

Kingsburgh, R. L., \& Barlow, M. J. 1994, MNRAS, 271, 257.

Kinney, A.L., Antonucci, R. R.J., Ward, M.J., Wilson, A.S., \& Whittle, M. 1991, ApJ, 377, 100.

Koski, A.T. 1978, ApJ, 223, 56.

Kriss, G. A., Davidsen, A. F., Blair, W. P., Ferguson, H. C., \& Long, K.S. 1992, ApJ, 394, L37.

Kriss, G., et al. 1997, this volume.

Krolik, J.H., \& Begelman, M.C. 1986, ApJ, 308, L55.

Macchetto, F., Capetti, A., Sparks, W.B., Axon, D. J., \& Boksenberg, A. 1994, ApJ, 435, L15.

Morris, S., Ward, M. J., Whittle, M., Wilson, A.S., \& Taylor, K. 1985, MNRAS, 216, 193.

Morse, J. A., Raymond, J. C., \& Wilson, A.S. 1996, PASP, 108, 426.

Mulchaey, J.S. 1994, Ph.D. thesis (University of Maryland).

Mulchaey, J.S., Wilson, A.S., \& Tsvetanov, Z. 1996, ApJ, 467, 197. 
Mundell, C. G., Holloway, A. J., Pedlar, A., Meaburn, J., Kukula, M. J., \& Axon, D. J. 1995, MNRAS, 275, 67.

Osterbrock, D.E. 1993, ApJ, 404, 551.

Pedlar, A., Dyson, J. E., \& Unger, S. W. 1985, MNRAS, 214, 463.

Pedlar, A., et al. 1993, MNRAS, 263, 471.

Pogge, R.W. 1989, ApJ, 345, 730.

Pogge, R.W., \& De Robertis, M. M. 1995, ApJ, 451, 585.

Pogge, R.W. 1997, this volume.

Prieto, M. A., \& Freudling, W. 1993, ApJ, 418, 668.

Rees, M. J., Netzer, H., \& Ferland, G.J. 1989, ApJ, 347, 640.

Robinson, A., et al. 1994, A\&A, 291, 351.

Simpson, C.J., Wilson, A.S., Bower, G.A., Heckman, T.M., Krolik, J. H., \& Miley, G. K. 1996, ApJ, in press.

Storchi-Bergmann, T., Mulchaey, J.S., \& Wilson, A.S. 1992, ApJ, 395, L73.

Storchi-Bergmann, T., Wilson, A.S., \& Baldwin, J. A. 1992, ApJ, 396, 45.

Storchi-Bergmann, T., Wilson, A. S., Mulchaey, J.S., \& Binette, L. 1996, A\&A, $312,357$.

Sutherland, R. S., Bicknell, G.V., \& Dopita, M. A. 1993, ApJ, 414, 510.

Tadhunter, C. N., Robinson, A., \& Morganti, R. 1989, in Extranuclear Activity in Galaxies, eds. E.J.A. Meurs \& R.A.E. Fosbury (Garching bei München: ESO), p. 293.

Tadhunter, C. N., \& Tsvetanov, Z. I. 1989, Nature, 341, 422.

Taylor, D., Dyson, J. E., \& Axon, D. J. 1992, MNRAS, 255, 351.

Unger, S.W., Pedlar, A., Axon, D.J., Whittle, M., Meurs, E. J.A., \& Ward, M. J. 1987, MNRAS, 228, 671.

Viegas-Aldrovandi, S.M., \& Gruenwald, R.B. 1988, ApJ, 324, 683.

Viegas-Aldrovandi, S. M. 1988, ApJ, 330, L9.

Viegas, S. M., \& Prieto, A. 1992, MNRAS, 258, 483.

Whittle, M., Haniff, C. A., Ward, M. J., Meurs, E. J. A., Pedlar, A., Unger, S. W., Axon, D. J., \& Harrison, B. 1986, MNRAS, 222, 189.

Whittle, M., Pedlar, A., Meurs, E.J.A., Unger, S.W., Axon, D.J., \& Ward, M.J. 1988, ApJ, 326, 125.

Whittle, M. 1989, in Extranuclear Activity in Galaxies, eds. E. J. A. Meurs \& R. A. E. Fosbury (Garching bei München, ESO), p. 199.

Wilson, A.S., \& Ulvestad, J.S. 1983, ApJ, 275, 8.

Wilson, A.S., \& Ulvestad, J.S. 1987, ApJ, 319, 105.

Wilson, A.S., Ward, M. J., \& Haniff, C. A. 1988, ApJ, 334, 121.

Wilson, A.S. 1992, in Physics of Active Galactic Nuclei, eds. S. J. Wagner and W. J. Duschl (Berlin: Springer-Verlag), p. 307.

Wilson, A.S. 1996, Vistas in Astronomy, 40, No. 1, 63.

Wilson, A.S., Braatz, J.A., Heckman, T. M., Krolik, J. H., \& Miley, G. K. 1993, ApJ, 419, L61.

Wilson, A.S., \& Tsvetanov, Z.I. 1994, AJ, 107, 1227. 